\title{
Scintillation Characteristics of CsI Crystal Doped Br under Gamma and Alpha Particles Excitation
}

\author{
Maria da Conceição Costa Pereira, Tufic Madi Filho \\ Instituto de Pesquisas Energéticas e Nucleares-IPEN/CNEN-SP, São Paulo, Brazil \\ Email: macoper@ipen.br
}

Received 20 February 2014; revised 24 March 2014; accepted 9 April 2014

Copyright (C) 2014 by authors and Scientific Research Publishing Inc.

This work is licensed under the Creative Commons Attribution International License (CC BY). http://creativecommons.org/licenses/by/4.0/

(c) (i) Open Access

\section{Abstract}

Inorganic scintillators play an important role in detection and spectroscopy of gamma, $\mathrm{X}$-rays and alpha particles as well as neutrons and charged particles. Scintillation crystals based on cesium iodide (CsI) are matters with relatively low hygroscopicity, high atomic number, easy handling and low cost, characteristics that favor their use as radiation detectors. In this work, pure CsI crystal and bromine doped CsI crystals were grown using the Bridgman technique. The concentration of the bromine doping element (Br) was studied in the range of $1.5 \times 10^{-1} \mathrm{M}^{-10} 10^{-2} \mathrm{M}$. The distribution of the doping element in the crystalline volume was determined by neutron activation. The result obtained with neutron activation analysis (NAA) has found that the mean values of Br found in grown crystals are consistent with those introduced in salt CsI, showing the incorporation of $\mathrm{Br}$ in the matrix structure of CsI. The optical transmittance assays were performed at a wavelength range of $110 \mathrm{~nm}$ to $1100 \mathrm{~nm}$. Analyses were carried out to evaluate the developed scintillators, concerning alpha particles and gamma radiation.

\section{Keywords}

Crystal Growth, Scintillators, Luminescence, Radiation Detectors

\section{Introduction}

The development of new radiation detectors using scintillation crystals, which permits to increase the speed of response, the accuracy in dose and energy, and at the same time, feasibility to simplify and reduce costs in the production process is always needed. Scintillators are materials capable of producing light when ionizing radiation dissipates its energy in their midst [1]. 
Inorganic scintillators, are explored in the new fields as positron emission tomography (PET), computed X-ray tomography (CT), spatial physics and astronomy. In the many cases CsI:Tl and NaI:Tl conventional scintillators are used, while barium fluoride $(\mathrm{BaF} 2)$ and bismuth germinate $(\mathrm{BGO})$ scintillators are appropriate for PET. Inorganic scintillators are also used in high energy physics, for measurements of gamma energy and electrons/positrons in accelerators. A scintillation detector of large dimensions, named electromagnetic calorimeter of total absorption (EM), carries a lot of crystals, in some cases more than 104 crystals and total weight up to 10 ton. The NaI:Tl was the first cintilator material used in calorimeter (EM). Subsequently, CsI:Tl crystals were used and, recently, BGO scintillators have been used. BaF2 and pure CsI scintillators have been used in radiation detectors of small proportions in HEP and in experimental nuclear physics [2].

There is, hence, constant interest in finding new scintillator materials or improving the characteristics of known scintillators. CsI doped with $\mathrm{Tl}$ is one of the most widely used scintillators because of its high yield $(\sim 65000 \mathrm{ph} / \mathrm{MeV})$ [3]. However, their long decay time $(\sim 1300 \mathrm{~ns})$ reduces their application mainly for low count rate measurements [4]. In contrast, undoped CsI has a very shorter decay time $(\sim 10 \mathrm{~ns})$, but its scintillation efficiency is also largely reduced ( $200 \mathrm{ph} / \mathrm{MeV})$ [1] [5]. CsI doped with cesium bromide was proposed as a scintillator that combines advantages of the both above mentioned crystals. The advantages of CsI matrix are low hygroscopicity, high atomic numbers, easily handling and low material cost. The aim of the present study is to grow pure and doped crystals for comparison. The effect of the bromine distribution in the crystalline volume was evaluated and its applications as scintillation detector. The energy resolution of the undoped CsI crystal was compared with the bromine doped CsI crystals, under gamma and alpha particles excitations.

\section{Materials and Methods}

The pure CsI and those CsBr doped crystals were grown in accordance with the vertical Bridgman technique [6] [7].

The starting materials, CsI salt (99.99\%) and the salts used as dopant $\operatorname{CsBr}(99.999 \%)$ were analyzed by means of EDXRF analytical technique ( fluorescence spectrometer for X-ray dispersion energy ) in order to determine any traces of impurities present in such commercially available salts. The X-ray fluorescence spectroscopic technique is a very versatile instrument multielement analysis can be applied to solid and liquid samples. The analysis by X-ray fluorescence is measured based on the intensity of the characteristic X-rays emitted by the sample components chemicals.

The subsequent determination of bromine concentration as dopant in the CsI: $\mathrm{Br}$ crystals was performed by neutron activation analysis (NAA) [8]. The investigated dopant concentrations were $10^{-2} \mathrm{M}, 5 \times 10^{-2} \mathrm{M}, 10^{-1} \mathrm{M}$ e $1.5 \times 10^{-1} \mathrm{M}$. This method consists, essentially, of production of artificial radionuclide from steady elements by irradiation under neutron flow and measure of emitted gamma radiation, which is characteristic for each element. The sample was irradiated in the IEA-R1 Nuclear Reactor for 8 hours. The applied neutron flow was $10^{12}$ $\mathrm{cm}^{-2} \cdot \mathrm{s}^{-1}$. The sampling for characterization analysis was collected in intermediate sections of crystals (at the same distance of the growth start).

The dopant concentration profile in the CsI: $\mathrm{Br}$ crystal $\left(10^{-1} \mathrm{M}\right.$ nominal concentration) was determinated by the same method. The crystal sample was cut in 18 slices, $6 \mathrm{~mm}$ each, as showed in Figure 1. One gram of each slice was taken for this analysis.

The X-ray diffraction is a very useful tool in the study of materials. In this work the Laue technique was used. By this technique it is possible to make a qualitative analysis of the degree of crystallinity of the crystals obtained by observing the diffraction spots (presence or absence of deformation) in Figure Laue crystal photographed [9].

Samples of the CsI crystals Br, in various concentrations of the doping element and pure CsI, were prepared in $1 \mathrm{~mm}$ thickness slices, suitably polished and subjected to exposure with a beam of incident X-ray from a molybdenum tube $(20 \mathrm{~mA}, 40 \mathrm{kV})$, at a distance of $50 \mathrm{~mm}$. The exposure time was 30 minutes, thus obtaining the spectrum of Laue.

The optical transmission measurements were made in the CsI: $\mathrm{Br}$ and pure CsI crystals. This measurement was performed at room temperature, using a Shimadzu spectrophotometer with a wavelength varying in the range between 200 to $800 \mathrm{~nm}$.

To study the response to gamma radiation and alpha particles, the crystals were machined, polished and coupled directly to the bi-alkali photomultiplier tube (RCA 8575 model, 21-pin). Silicone grease (Dow Corn- 


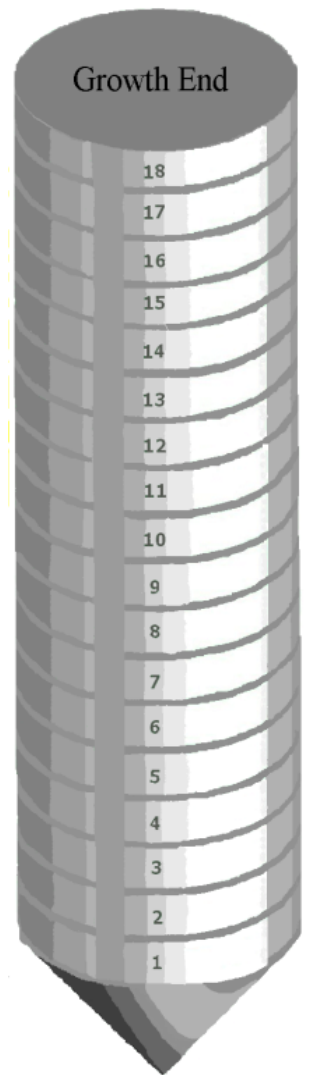

Start of the growth

Figure 1. Scheme of the CsI: $\mathrm{Br} 10^{-1} \mathrm{M}$ crystal.

ing-viscosity 1.0 McStokes) was the optical interface material. This provides a uniform refraction index at full contact surface between the crystal and the photomultiplier tube. The sides of the crystal which were not in contact with the photosensor were covered with Teflon tape to ensure good reflection of light. The radioactive sources were positioned in the center of the upper face of the crystal. The electronic modules used for processing signals from the photomultiplier tube were as it follows: pre-amplifier (Ortec model 276), amplifier (Ortec model 450), source voltage (Ortec 556), multichannel analyzer (Ortec ADCAM model 918A), Phillips oscilloscope (PM3295A 400MHz) and Pentium III microcomputer.

The energy resolution of the detectors crystal CsI: $\mathrm{Br}$ and pure CsI coupled to photomultiplier tube was determined using gamma radiation sources and energies between $355 \mathrm{keV}$ to $1333 \mathrm{keV}$, and $5.54 \mathrm{MeV}$ for alpha particles source. The operation voltage of photomultiplier was $2700 \mathrm{~V}$ for the detection of gamma rays and 2200 $\mathrm{V}$ for the detection of alpha particles. The accumulation time during the counting was $600 \mathrm{~s}$. The crystals used in gamma spectroscopy were cut with dimensions of $2 \mathrm{~cm}$ in diameter and $2 \mathrm{~cm}$ in height and in the spectroscopy for alpha radiation, crystals to $2 \mathrm{~cm}$ in diameter and $5 \mathrm{~mm}$ thick were used.

\section{Results and Discussion}

CsI: Br crystals, with $25 \mathrm{~mm}$ in diameter and $100 \mathrm{~mm}$ high were reproducibly grown by the Bridgman technique, at a concentration of $(\mathrm{Br})$ in the range of $1.5 \times 10^{-1} \mathrm{M}$ to $10^{-2} \mathrm{M}$. The crystals are shown in Figure 2(a) before heat treatment, Figure 2(b) after heat treatment and polish. Figure 2(b) is a photograph of the crystals on the description of its type in order to demonstrate its transparency.

Considering that impurities in the crystal lattice may affect the quality and characteristics photophysical of scintillator is necessary that the quality of the starting material is first considered. In Table 1 shows the concentration of trace impurities found in salt $\mathrm{CsI}$ and $\mathrm{CsBr}$ determined by fluorescence spectrometry X-ray energy dispersion. 


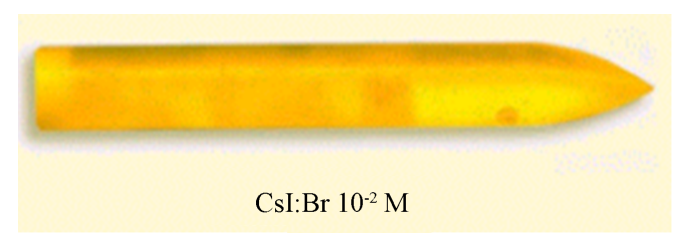

(a)

\section{CsI CsI:Br CsI:Br Csi Csi.jor CsI:Di CsI CsI:Br CsI:Br EsI Csl:B̈r CsI:Br}

(b)

Figure 2. Photos of crystals obtained. (a) before heat treatment; (b) after heat treatment and polish.

Table 1. Impurities found in salt $\mathrm{CsI}$ and $\mathrm{CsBr}$ determined by fluorescence spectrometry X-ray energy dispersion.

\begin{tabular}{ccc}
\hline Salt & Element & Concentration (ppm) \\
\hline Cesium iodide $(\mathrm{CsI})$ & $\mathrm{Zr}$ & $57 \pm 1.71$ \\
& $\mathrm{Fe}$ & $17 \pm 0.51$ \\
& $\mathrm{Zn}$ & $32 \pm 1.28$ \\
Cesium bromide $(\mathrm{CsBr})$ & $\mathrm{Ni}$ & $30 \pm 1.20$ \\
\hline
\end{tabular}

The dissociation of salts at high temperatures depends greatly on the purity of the starting materials used, so it is necessary that these materials are analyzed in advance. The experimental results determined by fluorescence spectrometry X-ray energy dispersion (Table 1), it it was not detected elements in the salts used in crystals growth, in concentrations that could be sources of contamination or be responsible by changes in the growth of crystals, confirming the quality of the salts, commercially available from Merck S/A.

In this work, the bromine was added to the starting material (salt CsI) with molar fractions of $10^{-2}, 5 \times 10^{-2}$, $10^{-1}, 1.5 \times 10^{-1}$. The results of neutron activation analysis represent the concentration of bromine in the crystal CsI: $\mathrm{Br}$ and pure CsI grown. They are shown in Table 2.

The obtained results by neutron activation analysis (NAA), Table 2, confirm that the average values found in the grown crystals are consistent with those introduced in the salt CsI, showing the incorporation of $\mathrm{Br}$ in the structure of the CsI matrix.

The concentration of the dopant in the crystal is an important parameter of quality control in the production of scintillator crystals, because the luminescence properties are affected by the dopant concentration. The results of the concentration of bromine in eighteen regions of the block of crystalline CsI: $\mathrm{Br} 10^{-1} \mathrm{M}$ after heat treatment are shown in Table 3 and shown in Figure 3.

The concentration of dopant in the crystal is a parameter of important quality control in the production of scintillators crystal, because of the luminescence properties are affected by the concentration of dopant.

In the study of distribution of the bromine dopant along the crystalline volume, bromine was added to the starting material (salt CsI) with mole fraction of $10^{-1}$. During the crystallization process, that concentration was changed resulting in a concentration gradient. The neutron activation analysis results indicated a higher concentration at the top of the crystal, with subsequent decrease in the initial phase of growth. A relative uniformity of Br concentration between slice 14 and slice 3 was found, as shown in Figure 3 and Table 3, which is the region of the crystalline volume indicated for use as radiation detector. From the slice 14 on, the concentration of bromine increases abruptly. Therefore crystals cutted in this region do not have uniformity of doping. The high concentration of bromine in the top of the crystal is expected, taking into account that the crystal grows from the bottom up and impurities tend to deposit at the end of the growth, due to the phenomenon of segregation.

A homogeneous distribution of dopants in a significant area of the crystals is highly desirable for materials for the construction of the radiation detector, since the optimization of the efficiency of light emission is dependent scintillation this homogeneity. However, the difficulty of obtaining uniform doping concentration in the direction of growth is due to the fact that, generally the distribution coefficient is different from the unit, leading to a concentration gradient.

The control distribution of dopants throughout the crystals is related to the phenomenon of segregation that occurs during the crystal growth. The micro-segregation is governed by localized perturbations at the solid-liquid interface and interferes with microscopic distribution of dopants in the crystals. These disturbances are represented by the effective distribution coefficient $\mathrm{k}_{\mathrm{ef}}$ which is directly related to the growth rate of the solid relative to the liquid. 


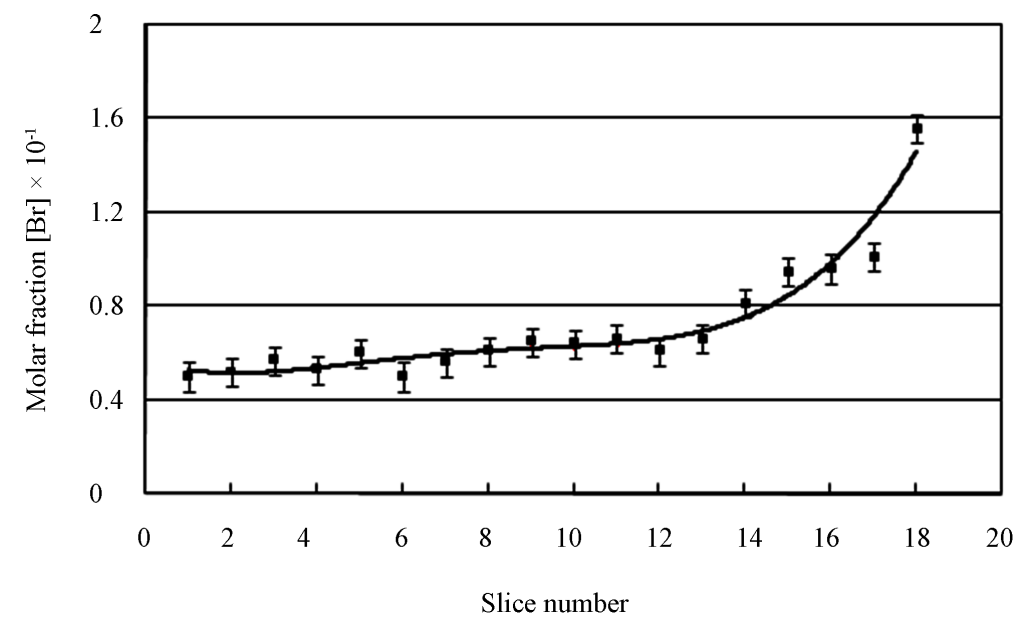

Figure 3. Concentration of bromine according to the height of the CsI: Br crystal.

Table 2. Content of bromine found in an intermediate region of the crystals CsI: Br, determined by neutron activation. Each result is the average of 3 results, with 3 different patterns of $\mathrm{Br}$ with the standard deviation.

\begin{tabular}{cc}
\hline Sampling of the crystals & Bromine concentration ( molar fraction) \\
\hline CsI:Br $10^{-2} \mathrm{M}$ & $0.75 \times 10^{-2} \pm 0.02 \times 10^{-2}$ \\
CsI:Br $5 \times 10^{-2} \mathrm{M}$ & $4.32 \times 10^{-2} \pm 0.12 \times 10^{-2}$ \\
CsI:Br $10^{-1} \mathrm{M}$ & $0.91 \times 10^{-1} \pm 0.02 \times 10^{-1}$ \\
CsI:Br $1.5 \times 10^{-1} \mathrm{M}$ & $1.41 \times 10^{-1} \pm 0.04 \times 10^{-1}$ \\
pure CsI & $\mathrm{ND}$ \\
\hline
\end{tabular}

ND-not detected under the experimental conditions of analysis.

Table 3. Determination of bromine concentration in 18 slices of $6 \mathrm{~mm}$ thick CsI: Br crystal, performed by neutron activation analysis.

\begin{tabular}{cc}
\hline Slice of the CsI: Br crystal & Bromine concentration ( molar fraction) \\
\hline 1 & $0.50 \times 10^{-1} \pm 0.003$ \\
3 & $0.52 \times 10^{-1} \pm 0.003$ \\
4 & $0.57 \times 10^{-1} \pm 0.004$ \\
5 & $0.53 \times 10^{-1} \pm 0.003$ \\
6 & $0.60 \times 10^{-1} \pm 0.004$ \\
7 & $0.50 \times 10^{-1} \pm 0.003$ \\
8 & $0.56 \times 10^{-1} \pm 0.004$ \\
9 & $0.61 \times 10^{-1} \pm 0.004$ \\
10 & $0.65 \times 10^{-1} \pm 0.004$ \\
11 & $0.64 \times 10^{-1} \pm 0.004$ \\
12 & $0.66 \times 10^{-1} \pm 0.004$ \\
14 & $0.61 \times 10^{-1} \pm 0.004$ \\
15 & $0.66 \times 10^{-1} \pm 0.005$ \\
16 & $0.81 \times 10^{-1} \pm 0.006$ \\
17 & $0.95 \times 10^{-1} \pm 0.007$ \\
18 & $0.96 \times 10^{-1} \pm 0.007$ \\
\hline & $1.01 \times 10^{-1} \pm 0.008$ \\
\hline
\end{tabular}


The results of Laue diagram indicate that the materials obtained are summarized in monocrystalline form. In Figure 4 and Figure 5 are shown the spectra obtained for the Laue crystal of pure CsI and CsI: Br, respectively.

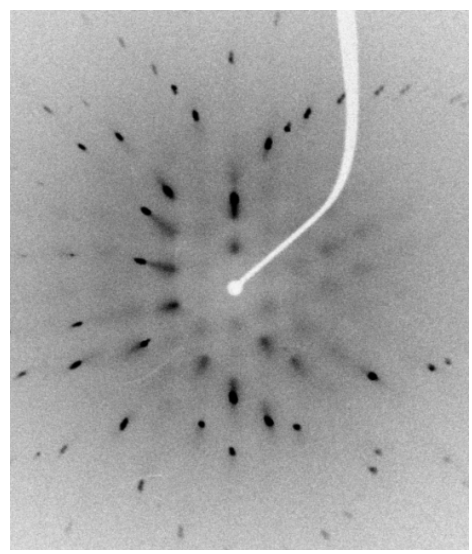

Figure 4. Laue diagram obtained for the pure CsI crystal.

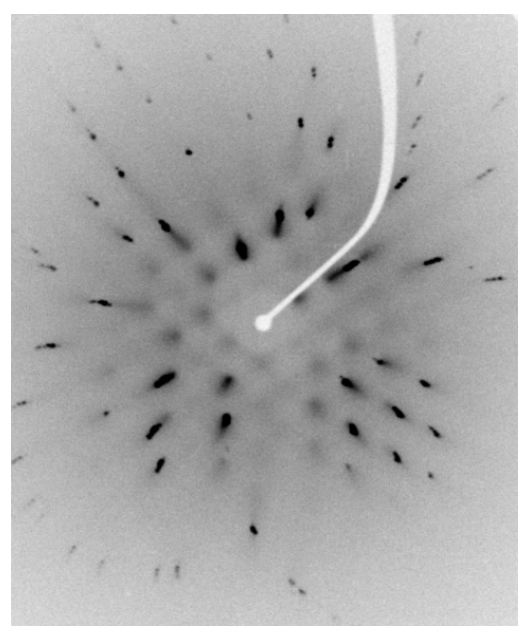

(a)

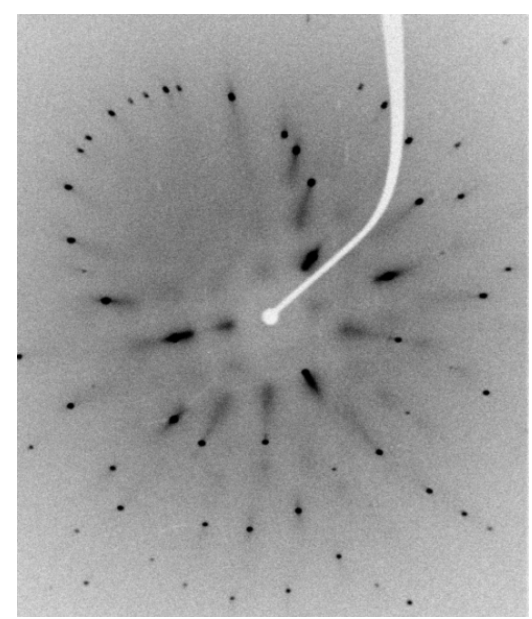

(c)

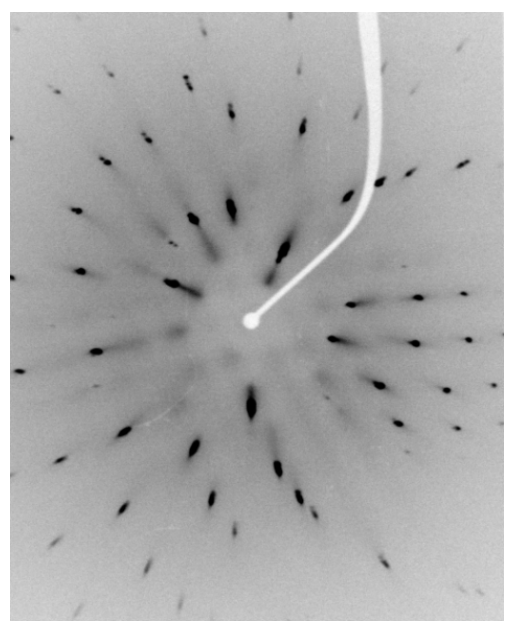

(b)

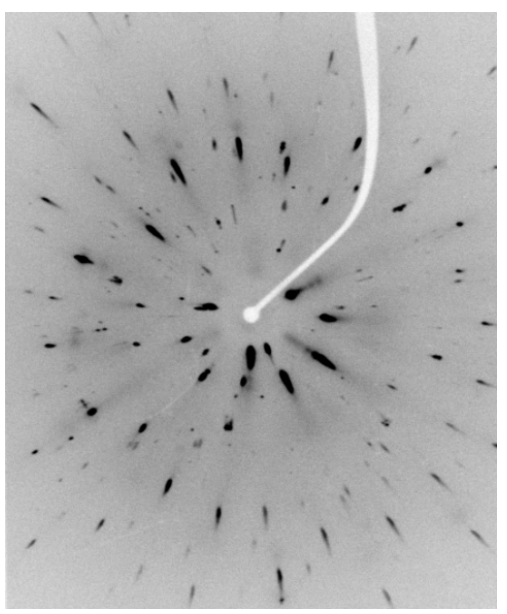

(d)

Figure 5. Laue diagram obtained for the crystal $\mathrm{CsI}$ : $\mathrm{Br}$ in various concentrations of the dopant element. (a) $10^{-2} \mathrm{M}$, (b) $5 \times 10^{-2} \mathrm{M}$, (c) $10^{-1} \mathrm{M}$, (d) $1.5 \times 10^{-1} \mathrm{M}$. 
Figures Laue (Figure 4 and Figure 5) have singular points, which indicate that these materials were pure CsI and CsI: Br single crystal structures match. In the case of CsI: Br probably due to replacement of the iodide ion $\left(\mathrm{I}^{-}=2.20 \AA\right)$ by ion bromide $\left(\mathrm{Br}^{-}=1.96 \AA\right)$ may have caused a little tension/distortion in the network due to the fact that the substitutional ion have a radius a slightly smaller than the ionic radius of iodide ion, represented in figure Laue by the presence of small spots on the different points of diffraction. Finally Laue spectra indicated that single crystals of good quality were obtained.

To quantify the degree of transparency of the produced crystals scintillators, optical transmittance tests were carried out in the range of luminescence. In all samples, the transmittance of the crystals notably decreased due to the decrease of the wavelength, Figure 6. Greater than $70 \%$ transmittance was observed for wavelengths above $400 \mathrm{~nm}$, which is the sensing area of the quantum efficiency of the photomultiplier tubes and photodiodes.

A significant decrease in transmittance compared to pure CsI crystal with increasing the concentration of bromine in the grown crystals. The decrease in optical transmittance curve of $\mathrm{CsI}$ : $\mathrm{Br}$, can be attributed to absorbance introduced into the crystal lattice $\mathrm{Br}$.

For the detection system crystals were coupled optically to the photocathode. The bright photons move the electrons of photocathodes and these are accelerated by a series of electrodes (dynodes) inside the photomultiplier tube. In the scintillation detection process, the proportionality is maintained in each step, that is, the number of photons released by the crystal is proportional to the energy released in crystal, from the incident radiation. The number of displaced electrons in the photocathode is proportional to the number of light photons from the crystal and the electric current generated by the photomultiplier tube. This way the height of the electrical pulse from the photomultiplier tube is proportional to the radiation energy absorbed by the crystal. This allows that the energy from different radionuclides can be distinguished from each other by pulse height. This also helps differentiate primary photons from photons that have been scattered by Compton effect before being detected. The photons that suffer Compton scattering have less energy than primary photons thus have lower pulse height.

Measures pulse height using gamma radiation sources of ${ }^{54} \mathrm{Mn}(835 \mathrm{keV})$ and ${ }^{137} \mathrm{Cs}(662 \mathrm{keV})$, Figure 7 and Figure 8 showed that the crystal CsI: $\mathrm{Br} 10^{-1} \mathrm{M}$ had the best time pulse and consequently the scoring range in the range studied energy when compared to pure CsI crystal and other crystals with dopant concentrations of the element bromine.

Each crystal scintillator developed and well characterized features ideal dopant concentration. According to Kaufman et al. [10] the ideal dopant concentration in a sodium iodide crystal doped with thallium (NaI:Tl) is about $0.1 \mathrm{~mol} \%$. Real above and below this value mainly concentrations cause a decrease in pulse amplitude. The findings of this study with respect to the pulse height are consistent with the results of these authors. Thus, the range of concentrations of bromine element studied, the best response with respect to pulse height was found for the crystal CsI: $\mathrm{Br} 10^{-1} \mathrm{M}$. For lower concentrations and for a concentration of $1.5 \times 10^{-1} \mathrm{M}$ was obtained in the diminution of pulse height. Therefore it is reasonable to indicate the concentration of $10^{-1} \mathrm{M}$ of bromine as the

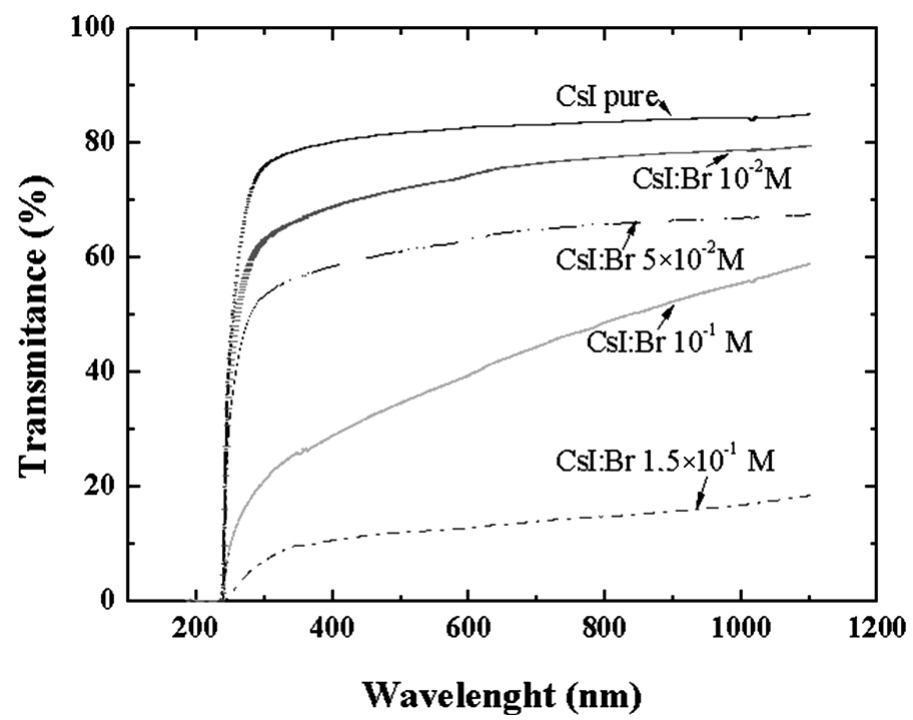

Figure 6. Transmittance curves of the CsI: $\mathrm{Br}$ at different concentrations. 


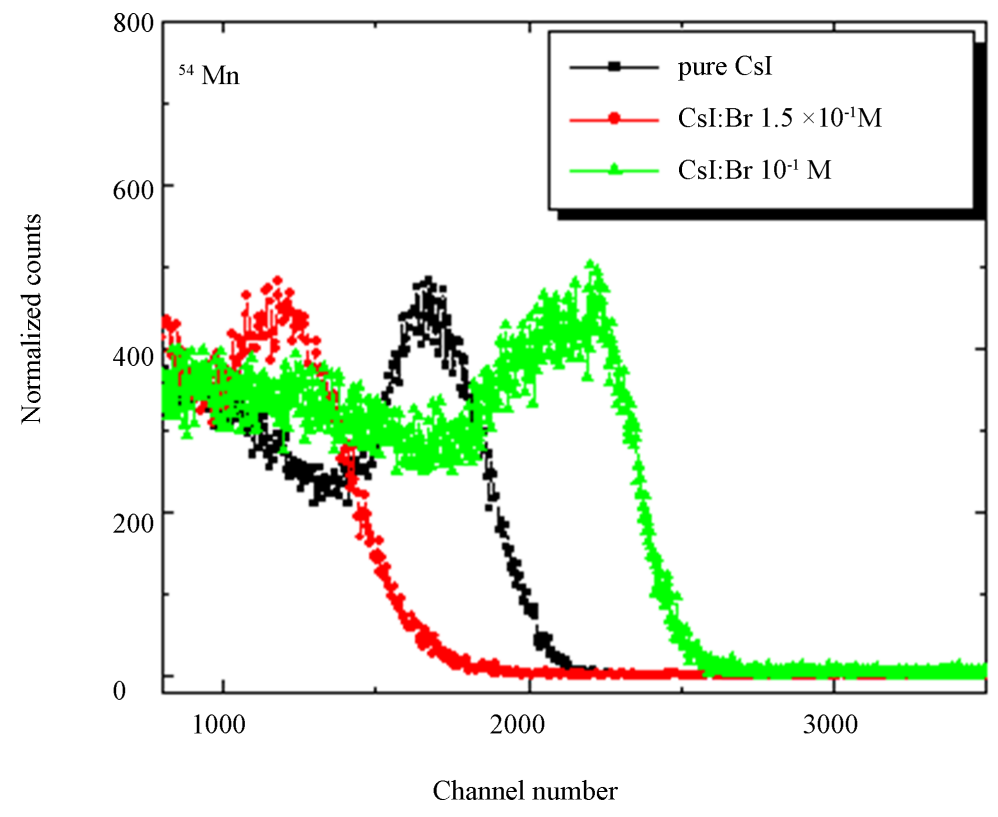

Figure 7. Pulse height obtained for gamma radiation from ${ }^{54} \mathrm{Mn}(835 \mathrm{keV})$ source with crystals of CsI: $\mathrm{Br}$ and pure CsI.

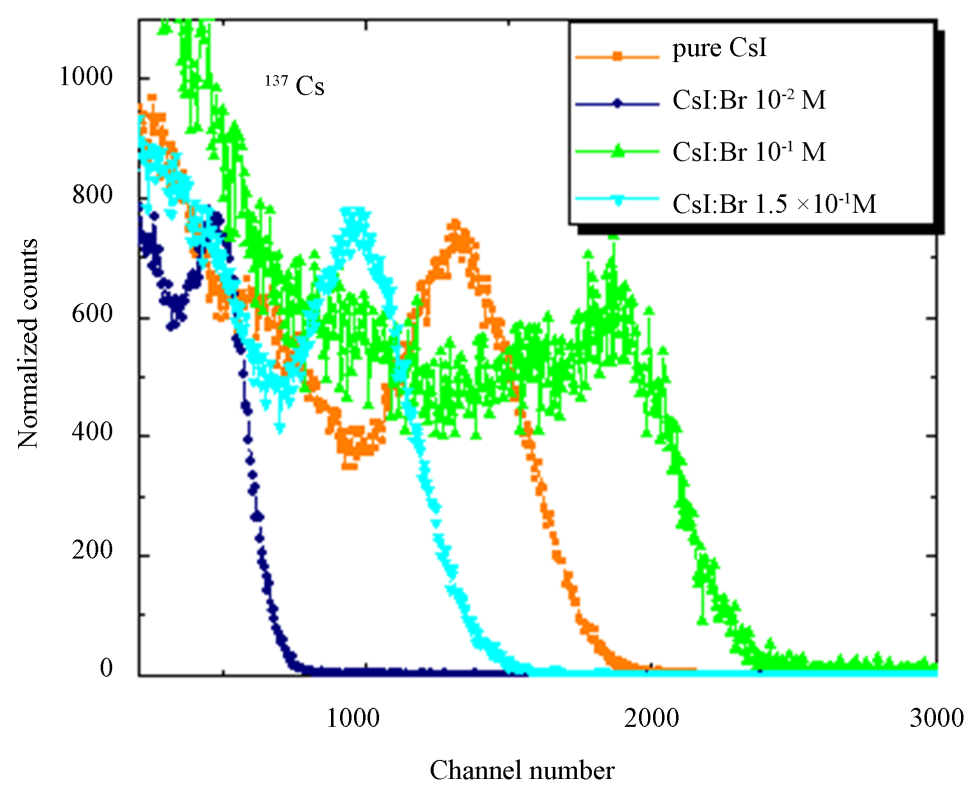

Figure 8. Pulse height obtained for gamma radiation from ${ }^{137} \mathrm{Cs}(662 \mathrm{keV})$ source with crystals CsI: $\mathrm{Br}$ and pure CsI.

best amount of dopant to be added to the CsI matrix.

The Figure 9 show alpha spectroscopy results for radiation of ${ }^{241} \mathrm{Am}(5.54 \mathrm{MeV})$ obtained with CsI: $\mathrm{Br}$ and pure CsI crystals.

For the evaluation of pulse height spectra obtained for CsI: Br when excited with gamma radiation it was found that the response to radiation of these crystals, in terms of pulse height, is dependent on the concentration of the dopant. However for alpha particle excitation, no significant differences were observed in the results of pulse height as a function of $\mathrm{Br}$ dopant concentrations. Best energy resolution results were found for the crystals, when excited with alpha particles compared to the results obtained with those excited with gamma radiation. We 


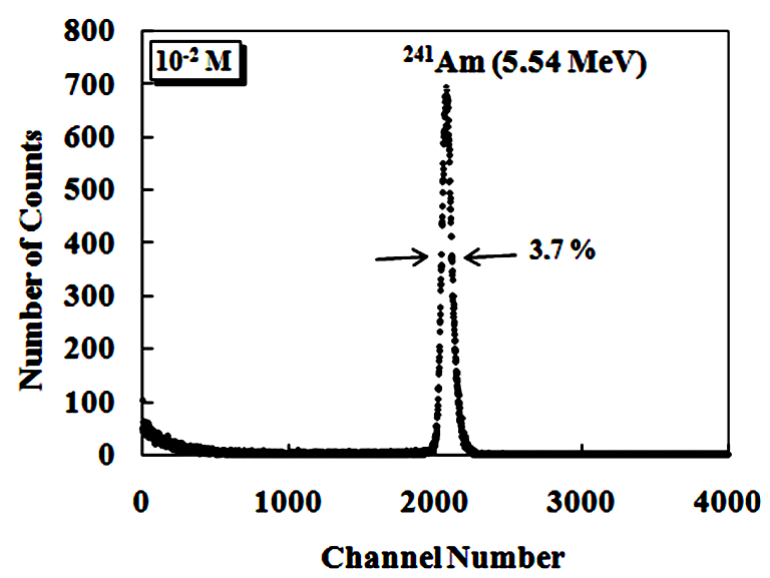

(a)

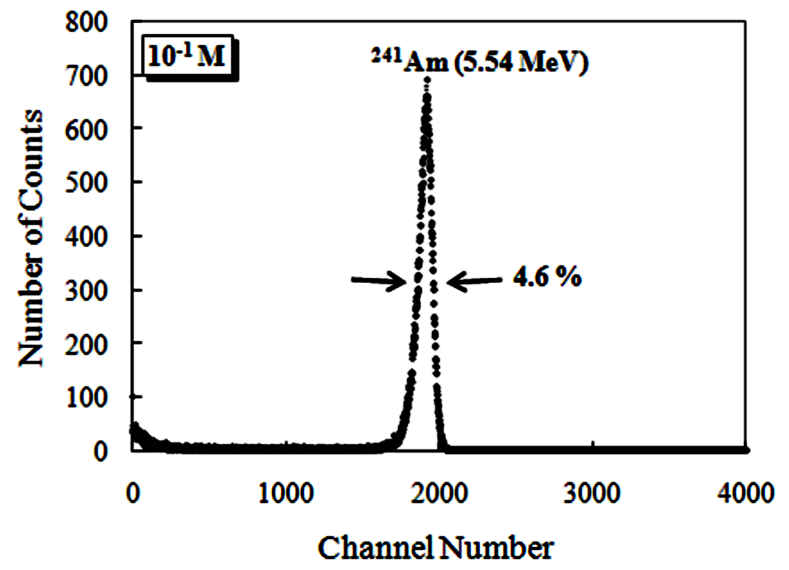

(c)

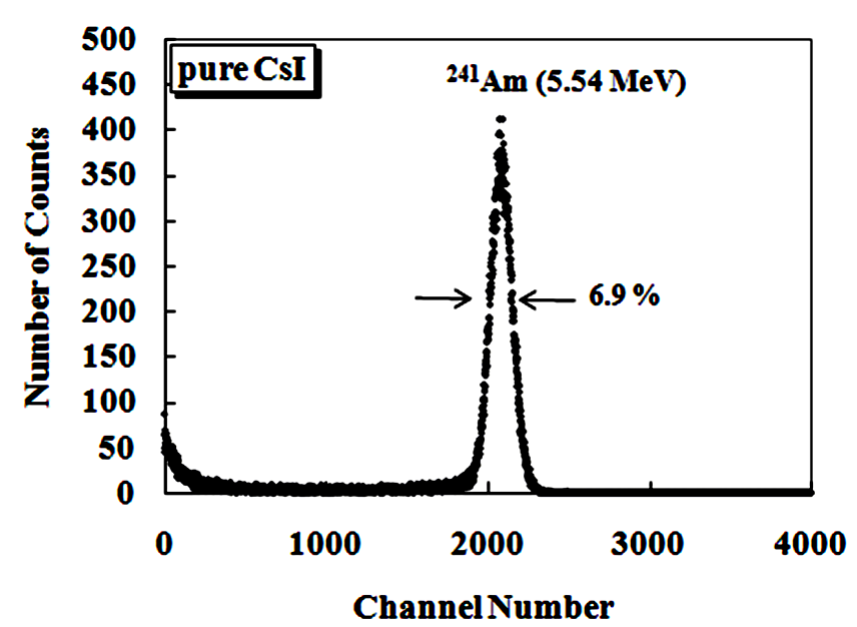

(e)

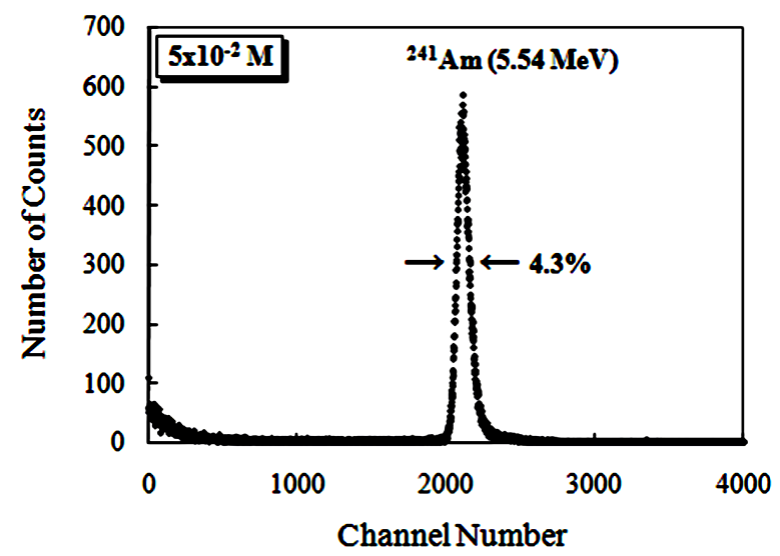

(b)

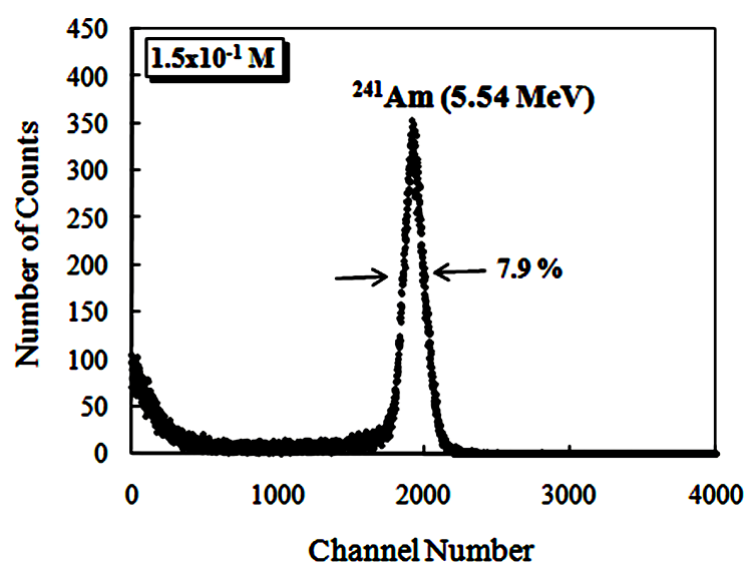

(d)

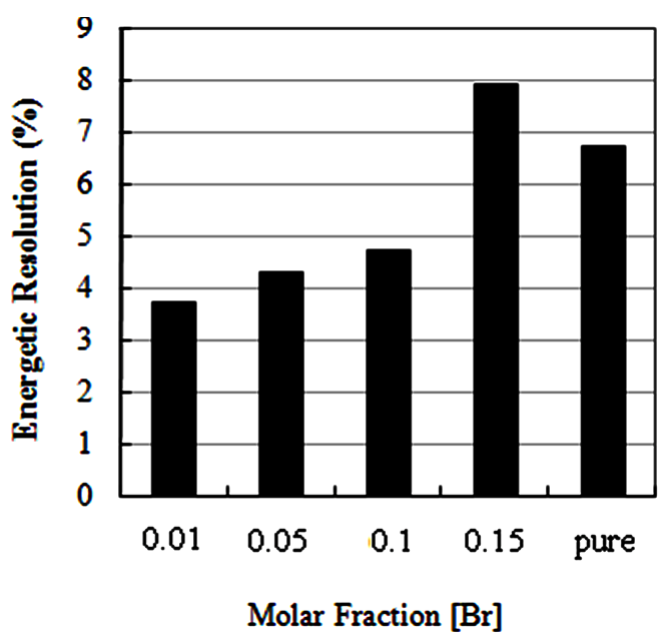

(f)

Figure 9. Energy spectra of the scintillation light from ${ }^{241} \mathrm{Am}(5.54 \mathrm{MeV})$ alpha particles in CsI: Br and pure CsI crystals. (a) CsI: Br crystal with $\mathrm{Br}$ concentration $10^{-2} \mathrm{M}$; (b) CsI: Br cystal with $\mathrm{Br}$ concentration $5 \times 10^{-2} \mathrm{M}$; (c) CsI: $\mathrm{Br}$ crystal with $\mathrm{Br}$ concentration $10^{-1} \mathrm{M}$; (d) CsI:Br crystal with Br concentration $1.5 \times 10^{-1} \mathrm{M}$; (e) pure CsI crystal; (f) Percent resolution of the $\mathrm{CsI}$ : $\mathrm{Br}$ and pure $\mathrm{CsI}$ crystals for the alpha radiation from the ${ }^{241} \mathrm{Am}$ depending on the concentration of the bromine dopant.

also observed an improvement in the energy resolution results of crystals of CsI: Br with decreasing concentration of bromine when excited by alpha particles. 


\section{Conclusions}

The vertical Bridgman technique used in this work for the growth of crystals CsI: Br and pure CsI proved to be quite adequate. The crystal growth process occurred in sealed crucible, permitting a more control the loss of volatile dopants (such as bromine) and control the purity of the obtained crystals.

The analysis of the bromine dopant concentration done by neutron activation showed the stability of bromine incorporated into the CsI structure. Analysis of bromine dopant concentration in eighteen pieces of crystal with $10^{-1}$ molar fraction showed that $70 \%$ of the crystal presented a relatively homogeneous region as concentration of bromine, which is the crystalline volume fraction suitable for use as scintillator.

The CsI: Br and pure CsI crystals showed optical qualities appropriate. The crystals grown in higher concentrations showed a significant decrease in the value of transmittance with increasing the concentration of the dopant element.

Laue figures showed characteristics points, which indicate that materials obtained CsI: Br and pure CsI single crystals structures correspond to good quality.

The CsI: Br crystals showed response to gamma radiation with defined photopeak spectra, and in the studied concentration range the crystal with molar fraction $10^{-1}$, presented the best resolution.

The results for the crystal CsI: Br with $10^{-1}$ molar fraction suggest uniformity of scintillation properties in the crystalline volume, probably due to adequate transparency in the region of luminescence, the emission of the activator and uniform distribution of individual emission centers, responsible by the scintillation in the crystalline volume.

The light production was dependent on the concentration of dopant, when the crystals were excited with gamma radiation. However, regarding to dopant concentration dependence for excitation with alpha particles, no significant differences were observed.

The crystals CsI: Br showed response to radiation of alpha particles demonstrating spectra with defined peaks, and in the concentration range of studied doping, the crystal with molar fraction $10^{-2}$ exhibited the best resolution.

\section{References}

[1] Knol, G.F. (2010) Radiation Detection and Measurement. John Willey \& Sons, New York.

[2] Lecoq, P. and Mikhailin, V.V. (2000) Inorganic Scintillators and Their Applications. SCINT 99, Moscow State University, Moscow.

[3] Swiderski, L., Moszynski, M., Nassalski, A., Syntfel-Kazuch, A., Czarnacki, W., Klamra, W. and Koslov, V.A. (2008) Scintillation Properties of Undoped CsI and CsI doped with CsBr. IEEE Transactions on Nuclear Science, 55, 1241 1245. http://dx.doi.org/10.1109/TNS.2008.920430

[4] Novoyny, R. (2005) Inorganic Scintillators-A Basic Material for Instrumentation in Physics. Nuclear Instruments and Methods in Physics, 537, 1-5. http://dx.doi.org/10.1016/j.nima.2004.07.221

[5] Weber, M.J. (2002) Inorganic Scintillators: Today and Tomorrow. Journal of Luminescence, 100, 35-45. http://dx.doi.org/10.1016/S0022-2313(02)00423-4

[6] Bridgman, P.W. (1925) Certain Physical Properties of Single Crystals of Tungsten, Antimony, Bismuth, Tellurium, Zinc Ant Tin. Proceedings of the American Academy of Arts and Sciences, 60, 305-383.

[7] Margulies, M., Witomski, P. and Duffar, T. (2004) Optimization of the Bridgman Crystal Growth Process. Journal of Crystal Growth, 266, 175-181. http://dx.doi.org/10.1016/i.jcrysgro.2004.02.043

[8] Hamada, M.M., Oliveira, I.B., Armelin, M.J. and Mesquita, C.H. (2003) Trace Impurities Determined by Neutron Activation in $\mathrm{PbI}_{2}$ Crystal Semicondutor. Nuclear Instruments and Methods in Physics Research Section A, 505, 517-520. http://dx.doi.org/10.1016/S0168-9002(03)01136-7

[9] Mantilla, J., Brito, G.E.S., Haar, E. Sagredo, V. and Bindilatti, V. (2004) The structure of $Z_{1-x} M_{n x} I_{2} S_{4} C_{4} C_{y s t a l s}$ Grown by Chemical Vapour Phase Transport. Journal of Physics: Condensed Matter, 16, 3555-3562. http://dx.doi.org/10.1088/0953-8984/16/21/005

[10] Kaufman, R.G., Hadley, W.B. and Hersh, H.N. (1970) The Scintillation Mechanism in Thallium Doped Alkali Halides. IEEE Transactions on Nuclear Science, 17, 82-87. http://dx.doi.org/10.1109/TNS.1970.4325679 